\title{
Observation and simulation of a filament eruption associated with the contraction of the overlying coronal loops and the filament rotation
}

\author{
X. L. Yan ${ }^{1}$, Z. K. Xue ${ }^{1,2}$ and Z. X. Mei ${ }^{1}$ \\ ${ }^{1}$ Yunnan Astronomical Observatory, Chinese Academy of Sciences, Kunming 650011, China \\ email: yanxl@ynao.ac.cn \\ ${ }^{2}$ University of Chinese Academy of Sciences, Zhongguancun Beijing 100049, China
}

\begin{abstract}
By using the data of Solar Dynamics Observatory (SDO), we present a case study of the contraction of the overlying coronal loop and the rotation motion of a sigmoid filament on 2012 May 22. At the beginning of the filament eruption, the overlying coronal loop experienced a significant contraction. In the following, the filament started to rotate counterclockwise. We also carried the simulation to investigate the process of the filament eruption.
\end{abstract}

\section{Introduction}

The contraction of the magnetic loop associated with flares and flarelike events has been shown by a number of authors (Forbes \& Acton 1996; Švestka et al. 1997; Liu et al. 2010). The shrinkage of large-scale loop without flares was also reported by Wang et al. (1997) using data of the Yohkoh Soft X-ray telescope. Wang et al. (1997) suggested that the shrinkage is not an apparent motion, but a real contraction of the coronal loops as a result of the heating at the footpoints followed by gradual cooling. Hudson (2000) suggested that a magnetic implosion at preflare energy storage must occur simultaneously with coronal events such as flares or coronal mass ejections.

\section{Observation}

The Atmospheric Imaging Assembly (AIA; Lemen et al. 2012) on board Solar Dynamics Observatory (SDO) provides multiple simultaneous high resolution full-disk images of the corona and the transition region. The observational range of the AIA can extend to $1.3 R_{\odot}$. We analyzed the contraction process of the overlying coronal loop and the rotation of the filament by using the $171 \AA$ and $304 \AA$ images of the AIA, which have high temporal $(\sim 12 \mathrm{~s})$ and spatial resolution $\left(0.6^{\prime \prime}\right)$.

\section{Result}

The successive contraction of the coronal loops overlying the filament can be seen from a series of $171 \AA$ images before the filament eruption (see the white arrows in Fig. 1). The maximum contraction speed was $45 \mathrm{~km} / \mathrm{s}$. After the contraction of the overlying coronal loops, the left part of the filament began to rotate counter-clockwise seen from the $304 \AA$ observation (Fig. 2). Meanwhile, the counterclockwise rotation of the right foot of the filament was also observed during its eruption (Yan et al. 2013). We also carried out the simulation of filament eruption (Fig. 3). We started with a parameter set that is used 

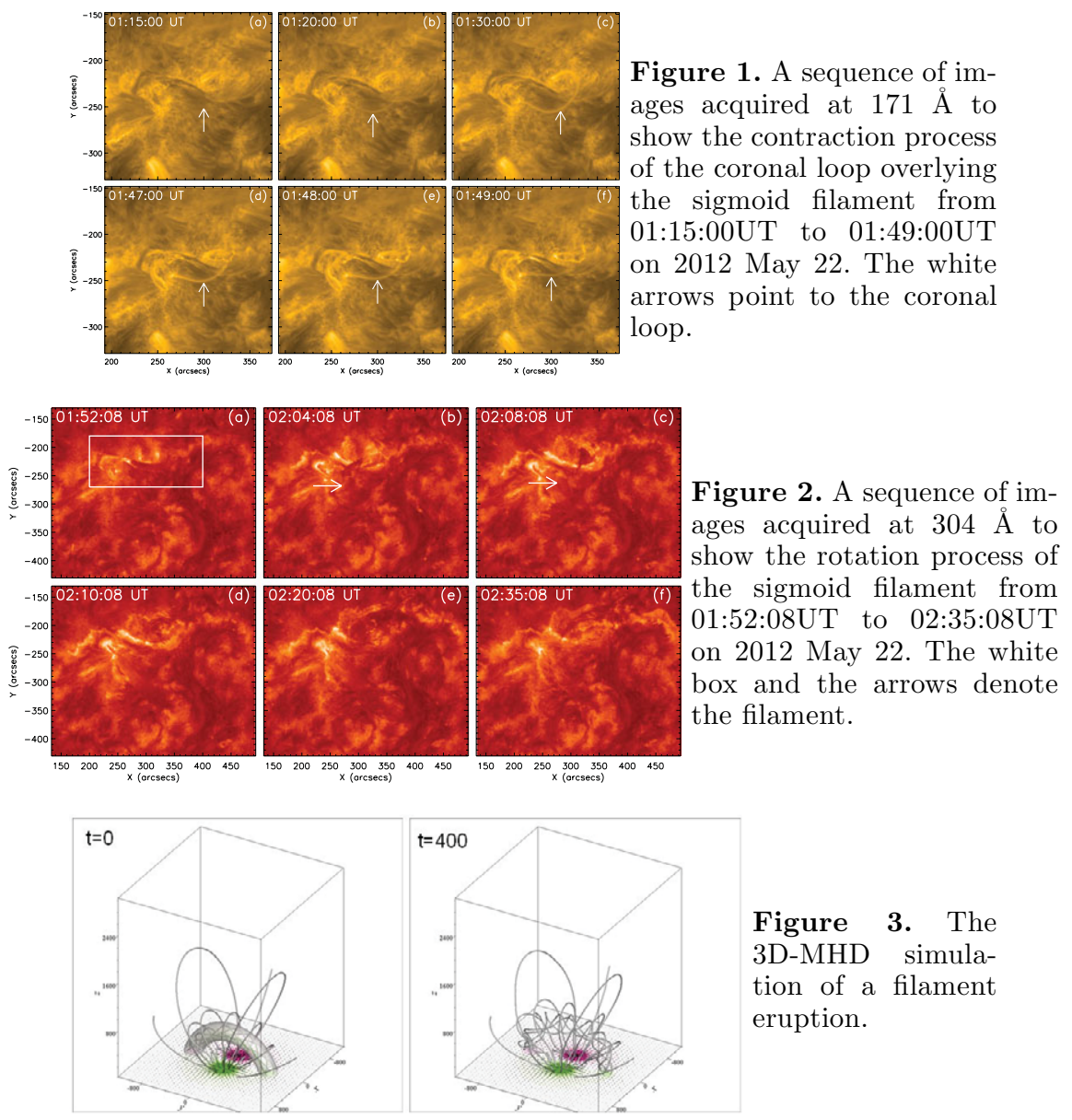

Figure 3. The 3D-MHD simulation of a filament eruption.

by Titov \& Demoulin (1999). The IRVANA code was used to carry out the simulation (Ziegler 2008). At the initial phase of filament eruption, we find that the coronal loop overlying the filament began to contract due to the expansion of the filament in our simulation. During the filament eruption, the overlying coronal loops at the end of the filament present the contraction as the coronal loops start to contract toward the below of the filament.

\section{Conclusion}

During the filament eruption, the contraction of the overlying coronal loops and the rotation motion of the filament was observed. Through the MHD simulation of filament eruption, the contraction of overlying coronal loop is found at the initial stage of the filament eruption. We explain that the contraction of the overlying coronal loops was due to the decrease of magnetic pressure and magnetic energy release. The obvious rotation of the right foot of the filament is caused by the unwinding motion of the filament during its eruption.

\section{Acknowledgements}

SDO is a mission of NASA's Living With a Star Program. This work is supported by the National Science Foundation of China (NSFC) under grant numbers 11373066, Key Laboratory of Solar Activity of CAS under number KLSA201303. 


\section{References}

Forbes T. G. 2000, J. Geophys. Res., 105, 23153

Hudson, H. S. 2000, ApJ (Letters), 531, L75

Liu, R. \& Wang, H. M. 2010, ApJ, 714, L41

Lemen, J. R., Title, A. M., Akin, D. J., Boerner, P. F., Chou, C., Drake, J. F., Duncan, D. W., et al. 2012, Solar Phys., 275, 17

Švestka, Z. F., Fontenla, J. M., Machado, M. E., Martin, S. F., \& Neidig, D. F. 1987, Solar Phys., 108, 273

Titov, V. S. \& Demoulin, P. 1999, A\& A, 351, 707

Wang, J., Shibata, K., Nitta, N., Slater, G. L., Savy, S. K., \& Ogawara, Y. 1997, ApJ (Letters), 478, L41

Yan, X. L., Pan, G. M., Liu, J. H., Qu, Z. Q., Xue, Z. K., et al. 2013, AJ, 145, 153

Ziegler, U. 2008, Computer Physics Communications, 179, 227 